\title{
Analysis of a survey of 36 French research committees on intracytoplasmic sperm injection
}

\author{
G. Moutel, N. Leroux , C. Hervé
}

Laboratoire d'Ethique médicale, de droit de la santé et de Santé publique, Faculté de médecine Necker, 156 rue de Vaugirard, 75015 Paris, France. (G Moutel MD, N Leroux, C Hervé PhD)

Biologie de la reproduction, CECOS, Centre Hospitalo-universitaire de Reims, Rue Cognacq-Jay, 51100 Reims, France. (G Moutel MD)

Correspondance to: Dr G. Moutel and Prof C. Hervé

Key Words: ICSI, biomedical research, Consultative Committees Protecting Participants in Biomedical Research (CCPPRB), law, medically assisted reproduction, biotechnologies, ethics. 


\section{Introduction}

ICSI (intracytoplasmic sperm injection) mechanically effects the introduction of the spermatozoon into the oocyte. The medical importance of this technique is considerable, as it lends itself to use in many cases of severe male hypofertility $(1,2)$. This new method allows for fertilizations to occur even in the case of sperm of very poor quality (quantitative and/or qualitative anomalies of spermatozoa). It thus allows the needs of many couples to be met, providing an alternative to seeking out a sperm donor. However, due to its still experimental character, ICSI raises a host of questions. Though traditional in vitro fertilization (IVF) provoked questions that are of an ethical nature $(3,4)$ (such as, transmission of a certain degree of infertility to the descendants in dysovulations or moderate cases of oligospermia with asthenospermia, idiopathic cases of infertility, and an increase in supernumerary embryos under care [5]), ICSI adds new ones (6-8): the consequences of intra-oocytal penetration, the act of injecting a spermatozoon that is naturally non-fertilizing and the genetic stock of which could be altered, the introduction of foreign matter into the heart of the oocyte, the fear of transmitting infertility to children born of this technique - all these innovations call for ethical interrogation (4).

As a recent report of the Academy of Medicine insists, these questions must be made the subject of a rigorous evaluation (9). Such an evaluation is all the more needed in that, alongside the indication of ICSI in cases of male infertility caused by very severe oligospermia with asthenospermia, one can envisage a proliferation of its indications; indeed, on account of the better performance (in terms of embryos and pregnancies produced) of ICSI over traditional IVF (1), we may well witness a rapid enlarging of the scope of ICSI's indication, to situations for which IVF would formerly have been recommended: less severe male infertilities, and, indeed, even female infertilities or infertilities that are idiopathic to a given couple.

Currently, the first results of ICSI indicate the birth of children without an increased frequency of clinical or caryotypical anomalies, leading some to say that it is legitimate to have given the green light to this new technology (10). It remains the case, however, that these studies have been contested (11); furthermore, the studies do not benefit from any considerable lapse of time since the implementation of ICSI. The majority of scientists call for vigilance (1-6) and rigorous evaluation, insisting on a familial genetic inquiry prior to the use of ICSI, as well as for a rigorous follow-up of pregnancies and births. Aside from the innocuous aspects of the technique, there remains over the long term a certain degree of uncertainty over the transmission of male infertility to the offspring in cases of severe male infertility (11).

It is worth noting the practical difficulty of setting up a long term follow-up of children born of ICSI, as well as the ethical difficulty of imposing such a follow-up on those subjects. 
The question arises as to the sorts of means that would allow for the tracking of the effects of this new technique within the framework of an epidemiological evaluation. Taken globally, these points call for a consideration of the modalities of informing couples and of obtaining their consent. Consent would have to follow a properly understood explanation of the uncertainties and advantages of the technique. The transmission of that information, which is essential to consent, calls professional responsibility into play. These elements also lead to the observation of the fact that, strictly speaking, the practice of ICSI continues to belong to the domain of experimentation (8), as it involves the application to human beings of a new biotechnology, the innocuousness of which is uncertain and is still in a process of being evaluated by professionals in new reproductive technologies.

In France, when a reproductive medical technique is first implemented, it must adhere to the rules defined by the 1994 Bioethics laws (12), which oblige centers to obtain ministerial authorization in order to operate; (This authorization is submitted first to review by the National Commission of Reproductive Medicine and Biology (CNMBR), established in 1995 $[12,13]$; the opinion of the CNMBR concerns one or more activities of medically assisted reproduction centers, and these centers have the obligation of presenting an annual report of activities). The Bioethics laws also impose an obligation of obtaining - prior to using any technique - the consent of the couple, after a full rendering of information followed by a one month period of reflection (12). Moreover, in France any new medical technology applied to humans, in conformity with the Huriet-Serusclat law concerning biomedical research (14), must be submitted to an evaluative protocol that has been put before one of the research committees, known as Consultative Committees Protecting Persons in Biomedical Research (CCPPRB).

The implementation of ICSI in France provokes debate over these different points. The aim of our work is to study the significance and the role of French research committees (CCPPRBs) in light of the implementation of ICSI.

\section{Method}

We presented each of France's 48 research committees (CCPPRB) with a national questionnaire aimed at evaluating the choices and decisional criteria that prevailed at the time ICSI was implemented in the different centers in each national region. Conceived in terms of narrow and open-ended questions, the questionnaire had a twofold goal: on the one hand, to study the modalities according to which protocols concerning ICSI were or were not submitted to CCPPRBs, and on the other hand to analyze the competency of the CCPPRBs themselves to deal with the new technique.

The survey was conducted from March to September 1996 (gathering of data), and from October 1996 to March 1997 (analysis of results). Responses to the narrow questions were 
studied in percentages, while responses to the more open-ended questions were analyzed for thematic content. The analyses were performed using Excel and Statview programs and the results are framed in terms of percentages of expressed responses.

\section{Content of the Questionnaire:}

1. Was a request for an opinion on the practice of ICSI submitted to the CCPPRB of your region? Yes, No. If "no," move on to questions 4 and 5. If "yes," questions 2, 3, and 5.

2. If yes. Did the CCPPRB declare itself competent to deal with the matter? Yes, No. If the CCPPRB declared itself incompetent, explain why.

Was the dossier passed on to another "ethical" authority? Yes, No. If yes, which one and what was its decision?

3. If your CCPPRB did in fact provide an opinion, was the opinion positive (authorization), negative (refusal), or conditional (authorization under certain conditions)?

What were the main difficulties you encountered in providing this opinion, and what recommendations accompanied your opinion?

4. If your CCPPRB was not consulted, are you aware if nonetheless in your region there are centers for medically assisted reproduction that practice ICSI? Yes, No, Don't know.

Do you think your CCPPRB should have been consulted? Yes, No, Why?

5. Do you think generally that ICSI comes under the application of the Huriet law and the affairs of a CCPPRB? Yes, No, Why?

Do you think another type of structure or another mode of regulation, other than CCPPRBs, is called for in such a case? Yes, No, Why?

\section{Results}

Of the 48 French CCPPRBs, 36 took part in this study. Among those 36, 23 CCPPRBs know that ICSI is being practiced in their region. Only 3 CCPPRBs $(8,3 \%)$, however, received dossiers concerning ICSI and gave an opinion.

One CCPPRB received two ICSI dossiers in 1993, and declared itself incompetent to deal with the matter. It then passed the dossier on to the National Consultative Ethics Committee (CCNE, the mission of which is to render nationally applicable decisions on ethical questions), which at the time did not follow up on the issue.

A second CCPPRB was also solicited in 1993, but since what they received was not a clearly defined research protocol, this CCPPRB returned the dossier to a local hospital ethics committee (in charge not of research issues but of ethical problems specific to the functioning of a hospital), which rendered a favourable opinion. 
In 1995, a third CCPPRB reviewed an ICSI submission: it deemed itself competent, though only after having consulted the National Consultative Ethics Committee (CCNE), which said that in this domain the CCPPRB was competent to deal with such a case. The CCPPRB then studied the dossier and rendered a positive opinion accompanied with advice and recommendations. This CCPPRB believes that supervision of the practice of ICSI was and remains essential, for a number of reasons: so that the technique will be approved only for specified and limited indications; so that very precise information be given to couples; and so there can be evaluation over the long and the short term. Its opinion thus reflected the recommendations of the CCNE (3):

- The need for couples to be provided clear and realistic information concerning both the experimental character of the method and possible alternative solutions to the problem of male infertility (adoption, sperm donor insemination).

- The need not to associate ICSI with other experimental practices or treatments.

- The requirement that the trial protocol be such that it allow for rigorous evaluation and a prospective analysis of the results. The results of the follow-up should, following the opinion of the CCNE, be transmitted to either the CCPPRB that had advised those who submitted the protocol or the CCNE itself.

- Finally, that everything be put into place so as to come up with an animal model, paralleling research being conducted on humans, that would help answer certain questions raised by the selection of different types of male gametes (mobile and immobile spermatozoa, spermatids...) and by the penetration of the oocyte.

In addition to these recommendations, the CCNE emphatically says that scientific responsibility is called for in the implementation of ICSI. When speaking of a couple's decision to undergo ICSI, the CCNE underscores the currently precarious ethical status of consent to ICSI, given the lack of certainty of the scientific data that can be transmitted and some couples' powerful desire for children, which can lead to a blind acceptance of risks.

As for the 33 CCPPRBs that received no ICSI submissions: 20 CCPPRBs $(60 \%$ of this group) are aware that ICSI is being used in their region, though no dossiers have been referred to them; 5 CCPPRBs responded that ICSI is not practiced in their region; 8 said they simply do not know.

7 CCPPRBs out of $33(21,2 \%)$ think that recourse to CCPPRBs was clearly necessary and that they were competent to manage such dossiers; 13 hold the opposite view; the remaining 13 gave no opinion on the matter.

Furthermore, out of the 36 CCPPRBs, $21(58,3 \%)$ feel that the implementation of ICSI could have come under the jurisdiction of CCPPRBs if it had been more clearly defined as part of their mandate. $27(75 \%)$ is the number of those that believe that other types of administrative bodies could have helped to regulate more coherently the establishment of a practice like ICSI, if necessary in partnership with the CCPPRBs, by giving a clear definition 
to the roles of each of the competent authorities: among the authorities mentioned were the Ministry of Health's National Commission of Reproductive Medicine and Biology (CNMBR, whose approval is a precondition for the delivery of ministerial authorization of the exercise by centers of any given technique), the National Consultative Ethics Committee (CCNE, the mission of which is to render decisions of national applicability on ethical questions, decisions which do not have the force of law), and local hospital ethics committees, which are not responsible for research issues.

Certain CCPPRBs make note of the fact that ICSI remains an experimental technique that, as such, does not offer the necessary distance in time to be considered a routine treatment. This for them implies the need for a protocol, accompanied by a CCPPRB's backing, especially regarding modalities of: informing the couple of risks and uncertainties; obtaining the couple's consent; evaluating the technique over the short and long term (development of pregnancies, pediatric follow-up of children, setting up tools and criteria for epidemiological measurement).

Other respondents discussed the modalities of recourse to a CCPPRB. It is their opinion that if the ethical principles set out in the Huriet law and the mandate of CCPPRBs apply to ICSI, then it would have been necessary - in view of the specificity of ICSI (considered as a procedure upon gametes and not living persons, though bringing a future life into play) - for the legislator or the ministerial authorities to clarify the role of CCPPRBs in this domain.

The issue of the protocolisation of ICSI was discussed by many of the CCPPRBs $(25 / 36$, $69,4 \%)$. They raised the question of whether the medically assisted reproduction professionals had or had not protocolised the implementation of ICSI in their centers, and whether they had or had not considered it as research. It may, indeed, be the case that at these centers no protocolisation had preceded the implementation of ICSI, which is considered by some to be routine medical treatment. A number of CCPPRBs pointed out a paradox in the law on biomedical research (14): if a clinician or researcher does not protocolise one of his/her scientific procedures and does not submit it to a CCPPRB, the latter has no mandate to detect such a procedure and to then bring it upon itself to review it.

Thus, a great number of CCPPRBs $(24,66,6 \%)$ believe that, independently of their own role, the evaluation procedures for ICSI should have been defined prior to any implementation of the technique, or any decision on the part of the centers to carry it out (even if this means perhaps officially putting the issue before a CCPPRB), by the relevant authorities within the Ministry of Health (General Health Directorate, National Commission of Reproductive Medicine and Biology [CNMBR], National Ministerial Committee for Sanitary and Social Organization), and by the scientific body itself. Judging from the fact that they were not consulted, certain CCPPRBs draw the conclusion that this reflects a general lack of debate prior to the implementation of a practice of great concern, as much for society as for its representative authorities. 


\section{Discussion}

The laws on bioethics of July 1994 (12), enacted contemporaneously with the emergence of ICSI, do not specifically mention the new technique. ICSI is one among other "techniques of medically assisted reproduction, requiring each center to obtain the approval and authorization of the Ministry of Health." When ICSI was being introduced, the medically assisted reproduction centers for the most part had to, and did, obtain approval for ICSI. These approvals were largely responsible for allowing the practice to get underway.

Without making a prejudgment as to the risks that may or may not be linked to ICSI, we observe from our results that this technique was mainly implemented without subjection to the rules of the Huriet law and of the committees (CCPPRBs) that supervise biomedical research in France.

The nature of ICSI merits being further specified, relative to its belonging to the realm of biomedical research. Should this reproductive technology be answerable to the ethical and legal rules drawn from the Huriet law (14) governing biomedical research in France? According to the terms of the Huriet law, "before conducting any project in biomedical research on a human being, a researcher must submit the project for review to the competent regional CCPPRB." This text furthermore calls not only for scientific prerequisites and standards but also for a preliminary assessment of the benefits and risks involved and of the uncertainty linked to the new practice. Following from these are the modalities of providing patients with information concerning benefits and risks, information that grounds the obtaining of their consent. The spirit of these rules is one that permits the establishment of research and evaluation procedures that are suitable, not only on the scientific level, but equally on the ethical level and on that of the acceptance of a practice by the entire community.

Our results show that neither application of the Huriet law nor recourse to the CCPPRBs were effectively brought into play at the time when ICSI was implemented. The problem this raises concerns the actual regulatory methods and structures that are currently in place to deal with the application of new biotechnologies to humans.

In the case of ICSI, one could argue that not involving a CCPPRB is explained by the nature of ICSI itself, which acts upon gametes and not, at first glance, upon living persons. However, this approach is reductionist. After all, gametes are the product of human bodies and cannot be made objects of materialization, especially since their manipulation involves a future living person. All this is concretized in the fact that ICSI achieves its results by means of two persons, a man and a woman, who together constitute the "parental couple" and whose decision implies, and impacts upon, the future child (over whom, as the Academy of Medicine emphasizes [9], there hovers some uncertainty). 
Furthermore, from the outset, ICSI could be categorized fully under the definition of "new technologies applicable to humans," and that being the case, it could have been subjected to the protocols of evaluation according to the modalities defined by the Huriet law. Its implementation could have been supervised by the CCPPRBs, which are authorities that are legally competent in the domain of biomedical research. The spirit of the Huriet law was clearly enunciated in J.F. Mattei's report: "the application of the law does not depend on the nature of the proposed product or technique; it aims to protect persons and to that end establishes rules that are to govern the implementation of any new technology among humans in general" (15).

Focusing on the example of ICSI, our study shows that, in fact, actual practices can and do skirt the law's collective ethical rules. A fundamental question thus arises concerning the role of CCPPRBs. It is worth recalling a limit of the Huriet law on research, namely that if a clinician or researcher does not protocolise one of his/her scientific procedures and does not submit it to a CCPPRB, the latter has no mandate to hunt out such an activity and to take it upon itself to sit in judgment of it.

We have thus to engage in inquiry in order to know how a posteriori, now that ICSI is practiced in numerous centers, it should adhere to principles of evaluation and safety already in existence for other medical technologies (new surgical technologies, hemodialysis, new kinds of medication, new kinds of biomaterial....) (15). According to article 10 of the Huriet law (14), these cautionary principles could have come from CCPPRBs if they had been consulted: "The CCPPRB can issue a judgment favourable to the conducting of a research project on condition that relevant information is transmitted throughout the project's duration." These terms are similar to those of recommendation n. 42 of the National Consultative Ethics Committee (CCNE), from March 1994: "the protocols of experiments concerning intracytoplasmic sperm injection - ICSI - must be such as to allow for a rigorous evaluation and a prospective analysis of results; the results of this follow-up must be transmitted to the CCPPRB that reviewed the protocol, or even to the CCNE itself" (3).

Given that the neither the CCPPRBs nor the CCNE were able to play this role, our results raise questions concerning the sorts of evaluation procedures that should today be put in place. Research into reproduction should not be challenged as such, but it is important that the society responsible for the implementation of new reproductive technologies be assured that these will be subject to adequate supervision and follow-up. Such supervision is inseparable from the exercise of collective responsibility over the short and long terms. The question that today arises is: given that ICSI is considered to be a therapeutic act that is fraught with a degree of uncertainty, how can its evaluation be carried out in a spirit of vigilant medically assisted reproduction? As was previously the case for traditional IVF and the freezing of embryos, ICSI cannot escape scrutiny merely because it is said to be too soon to know its eventual long-term effects. Criteria of good practices and of rigorous evaluations 
are tangible elements of a vigilance that needs to be implemented. Today, it seems necessary that independent regulatory organisms define collective, mandatory procedures for all centers authorized to practice ICSI. These procedures should follow the example of the rules already in use for other treatments that are carefully evaluated after they are brought to market (pharmaco-vigilance for medications, 'hemo-vigilance', follow-up of biomaterials, etc.).

It should be noted that our results indicate as well the necessity, when a new technique is implemented by professionals, of a clarification of the respective competencies and the articulations between: on the one hand, the CCPPRBs, which are responsible for biomedical research, and, on the other hand, the ministerial authorities in charge of approving and evaluating centers for medically assisted reproduction. One relatively simple idea could render the current situation more coherent. There should be an elaboration, alongside the ethical and scientific criteria in the Huriet law on biomedical research, of corresponding criteria that would be brought into play both in regulatory procedures governing the approval of centers for medically assisted reproduction and in the procedures for sanctioning the techniques and materials used. Among these procedures, a key role is assumed by the obligation to submit a request (to use a given procedure) for evaluation, an evaluation that must take into account the analysis of indications (16) (types of infertility), the results, costbenefit consequences, quality of information provided to couples, and psychological concerns. As for long term evaluation (future of children born, transmission of infertility, detection of other anomalies), it seems essential that centers for medically assisted reproduction allow for the epidemiological tracking of patients. This could be done by keeping a precise medical file on the parents and the child, though the modalities for managing this dossier over a long period of time are as yet undefined. These evaluation procedures taken all together could form the basis of the contract of approval authorizing the centers to practice ICSI.

Such an approach would allow for a clearer vision of the role of each player and an appreciation of a greater complementarity among them, in the eyes of scientists, patients and the public.

\section{Acknowledgments}

We thank Prof P Sado and the «Conférence nationale des comités de recherche français (CCPPRB) ». Whe also thank the following CCPPRB members involved in this evaluation: Alsace I Strasbourg (J.D. Tempé), Auvergne Clermont-Fd (A. Eschalier), Basse Normandie Caen (J. Leménager), Boulogne A. Paré (J.M. Le Parc), Bordeaux B (A. Taytard), Bourgogne Dijon (D. Lambert), Champagne-Ardennes Reims (C. Pourny), Franche Comté Besançon (P. Bechtel), Grenoble (L. Barret), Lorraine Metz (G. Jacques), Lorraine Nancy (P. Peton), Lyon Hotel Dieu (A. Nageotte), Lyon Léon Berard (J.L. Clément), Nice (S. Dumas), Nimes (J. Fourcade), Marseille I (F. Giraud), Marseille II (Y. Carcassonne), Montpellier St Eloi (M. Rodière), Paris Bichat Claude Bernard (A. Dauphin), Paris Bicètre (P. Duroux), Paris Cochin 
(G. Olivé), Paris Hotel Dieu (J.L. Selam), Paris Necker (M. Hamon, C. Hervé), Paris Pitié Salpétrière (M. Raphael), Paris Saint Louis (J. Rouffy), Pays de Loire I Anger (P. Pezard), Pays de Loire II Nantes (D. Duveau), Picardie Amiens (J. Descoubes, A. De Broca), Poitou Charentes Poitiers (B. Becq-Giraudon), Rennes (P. Sado), Rhone Alpes Loire Saint Etienne (J.M. Vergnon), Saint Germain en Laye (F. Beaufils), Toulouse I (J.P. Marc-Vergnes), Toulouse II (J. Montagut), Tours (M. Breteau), Versailles (N. Brion, P. Maroteaux). 


\section{References}

1 Van Steirteghem AC, Nagy Z, Joris H et al. High Fertilization and Implantation Rates after Intracytoplasmic Sperm Injection. Human Reprod 1993; 8: 1061-6.

2 Palermo GD, Cohen J, Rosenwaks Z. Intracytoplasmic Sperm Injection: a Powerful Tool to Overcome Fertilization Failure. Fertil Steril 1996; 65: 899-908.

3 French National Consultative Ethics Committee. Notice n. 42 of the French National Consultative Ethics Committee on the Development of Medically Assisted Reproduction Practices. Dictionnaire permanent bioethique et biotechnologies 1994; 9: 9793-95.

4 David G. Interrogations pour l'avenir de l'enfant. Bull. Acad. Natle. Med 1995; 179: 17511757.

5 Moutel G. Eléments d'évaluation du devenir des embryons surnuméraires; objectifs d'une évaluation éthique en santé publique. RBM (Revue européenne de biotechnologies médicales) 1995; 17: 101-106.

6 Liebaers I, Bonduelle M, Van Assche E, Devroey P, Van Steirteghem AC. Sex Chromosome Abnormalities after Intracytoplasmic Sperm Injection. Lancet 1995; 346: 1095.

7 Govaerts I, Englert Y, Vamos E, Rodesch F. Chromosome Abnormalities after Intracytoplasmic Sperm Injection. Lancet 1995; 346: 1095-1096.

8 Hervé C, Moutel G. Abnormalities after Intracytoplasmic Sperm Injection (Medical Ethics). Lancet 1995; 346: 1096-1097.

9 Laplane R, David G. Assistance médicale à la procréation: Problèmes actuels. Bull. Acad. Natle. Med 1996; 180: 11-30.

10 Liebaers I, Bonduelle M, Legein J, Wilikens A, Van Assche E, Buysse A, Wisanto A, Devroey P, Van Steirteghem AC. Follow-up of Children Born after Intracytoplamsic Sperm Injection. Fertil Steril, Proceedings of the 15th World Congress on Fertility and Sterility, Sept 1995, 409-412.

11 Moutel G, Hervé C, Tritto J, Boucaya V, Le Roux N. Injection intracytoplasmique de spermatozoïde: enjeux éthiques et évaluation. Press Med 1996; 25: 989-993.

12 Law n. 94- 654, of July 1994, Concerning Donors and Use of Products and Elements of the Human Body, Medically Assisted Reproduction, and Prenatal Diagnosis. JO République française 30 July 1994, 11060-11068.

13 Decree of 6 November 1995 directing for the nomination of members of the National Committee on Medical and Biological Reproduction and Prenatal Diagnosis. JO république française du 10 novembre 1995, 16648.

14 Huriet law n. 88-1138 of 20 December 1988 concerning the protection of persons lending themselves to biomedical researches; and law n. 94-630 of 25 July 1994, modifying the IIbis Book of the Code of Public Health, concerning the protection of persons lending themselves to biomedical researches. JO République française du 26 juillet 1994, 10747-10749. 
15 Mattei JF. Bilan de l'application de la loi Huriet. Rapport à monsieur le premier ministre sur l'éthique biomédicale en France. Ministry of Social Affairs, Ministry of Research and Higher Education, 1993, 49-69.

16 Moutel G, Hervé C, Adnet JJ. Has Traditional Sperm Analysis Lost its Clinical Relevance? Fertil Steril 1997; 67: 583-585. 